\title{
Atelier sur la gestion d'énergie autour de micro-générateurs
}

\author{
Bruno Allard, Jacques Verdier *
}

30 janvier 2015

\begin{abstract}
Résumé
La récupération d'énergie est déjà largement le cadre d'enseignement et de travaux pratiques au sein des formations utilisatrices des moyens du GIP CNFM. La gestion de l'énergie électrique, issue des micro-générateurs, l'est un peu moins. Afin de faire une sorte de synthèse entre le contenu pédagogique entendu sous le vocable « électronique de puissance » et celui associé à l'électronique basse-puissance, ou encore ce que revêt l'intégration microélectronique, il nous est apparu intéressant de proposer aux étudiants un atelier pour aborder par la pratique des concepts à travers des objets physiques manipulables (au sens de la robustesse, de la sécurité...). L'étudiant est mis en présence de «fonctions » afin de produire une solution d'alimentation autonome d'un capteur. L'étudiant a pour objectif de réfléchir à une solution, compte-tenu des spécificités d'un capteur donné, et de mesurer des performances énergétiques au sein du système d'alimentation autonome que l'étudiant aura construit. Le debriefing de l'atelier est le lieu d'une discussion plus technique et théorique sur certains aspects des "fonctions" que l'étudiant aura mises en oeuvre. Les exemples expérimentaux doivent servir ensuite de fil rouge dans certains cours.
\end{abstract}

\section{Introduction}

Le pôle de Lyon du GIP CNFM, accueilli au Département de Génie Electrique de l'INSA de Lyon, fournit des moyens à des formations autour des systèmes embarqués communicants. Les moyens microélectroniques affairant à la technologie sont

*Université de Lyon, INSA de Lyon, Pôle CNFM de Lyon (CIMIRLY) 
disponibles dans le pôle de Grenoble et à une moindre échelle sur Lyon. Le pôle de Lyon offre donc essentiellement des moyens logiciels et expérimentaux.

Une demande pédagogique, en 2008, a concerné la mesure de l'énergie consommée par un système embarqué pour mettre cette grandeur en relation avec l'activité logicielle. Cette demande a débouché sur une offre pédagogique présentée en 2010, et 2012, et largement utilisée maintenant [1].

Cette thématique, encore appelée OS-driven power management, se trouve à la convergence entre des problématiques matérielles circuits (régulateurs de tension monolithiques, VCO, RF-PA) et des problématiques logicielles (Event-driven, API). En gros, il s'agit de ralentir les services rapides quand l'application le permet, pour gagner en énergie à qualité de service égale. Ou bien d'utiliser les mémoires cache pour éviter de solliciter des mémoires plus lointaines, donc plus coûteuses en énergie d'accès. Cette idée trouve une concrétisation par une technique appelée Digital Voltage and Frequency Scaling (DVFS). Seul l'OS est en mesure de savoir si un service peut être ralenti. Il y a bien sûr des mécanismes à mettre en œuvre pour compléter un logiciel des informations permettant la mise en œuvre de la régulation de tension et de réglage de la fréquence d'horloge. Cette approche est un excellent moyen de rapprocher des publics d'étudiants issus de cursus différents : à savoir informatique, télécommunication et électronique. Outre les aspects techniques de mise en oeuvre de travaux pratiques sur la manipulation de l'énergie en lien avec du logiciel, l'expérience a permis de comprendre comment les cours connexes dans les formations utilisatrices pouvaient d'approprier le vécu des étudiants dans les travaux pratiques qui ne concernaient pas directement les cours.

Les équipes enseignantes peuvent relayer l'information mais le déroulement des formations, avec le grand nombre d'intervenants, reste une juxtaposition d'évènements pédagogiques pour les étudiants, sans réelle opportunité de formalisation de la cohérence. L'expérience acquise sur les travaux pratiques à l'échelle énergie/logiciel a mis en évidence qu'un détail technique mis en oeuvre en séance pratique mais non complètement expliqué, resurgissait toujours dans un cours connexe. Une possibilité est donc de mettre les étudiants en face de la liste de ces détails un peu masqués par l'activité pratique, pour les enjoindre à les travailler par eux-mêmes ou bien rebondir à bon escient quand l'occasion de présenterait dans les cours concernés. Cette liste ne peut pas surgir de nulle part car l'étudiant doit être persuadé de son bien-fondé. L'approche logique est donc celle d'un debriefing après travaux pratique. Il s'est donc imposé l'idée de transformer certains TPs plutôt en atelier dès lors que l'étudiant doit appréhender les problématiques d'un système plutôt que d'une question en particulier. L'étudiant met en application une méthodologie donnée dans un cadre technique donné, pour acquérir du savoir-faire pratique (mais là n'est pas l'essentiel) et surtout 
appréhender lui-même la cohérence d'un ensemble de détails pour observer les performances à l'échelle du système et ensuite s'interroger sur chacun des détails (après l'atelier). A l'enseignant de mettre l'étudiant en face des éléments les plus pertinents et lui fournir les informations nécessaires pour auto-apprendre ou rebondir dans un autre cours, travaux dirigés ou travaux pratiques. Voilà pour le cadre.

Les TPs sur les « systèmes embarqués communicants », essentiellement pris entre les problématiques d'OS, de code, de profilage, de gestion de tension plus ou moins rapide, ont soulevé naturellement la question de l'autonomie énergétique à partir de récupération d'énergie ambiante. L'ère de l'Internet des Objets et la prolifération des objets connectés, renforce cette question technique. La récupération d'énergie reçoit un écho très important en recherche. Les principes physiques sont déjà illustrés en enseignement (photovoltaïque, effet Seebeck, piézoelectricité...). Des ouvrages de vulgarisation sont même déjà disponibles. Nous avons proposé de nous intéresser aux cas de récupérations de l'énergie dans des contextes moins connus ou plus difficiles, c'est-à-dire dans le cas de disponibilité difficile et/ou de densité d'énergie très faible. La littérature parle de micro-générateurs pour qualifier cette partie d'un système relatif à l'approvisionnement en énergie. Au-delà du micro-générateur et des principes physiques, les compétences présentes sur le Pôle de Lyon du GIP CNFM vont vers la gestion de ces énergies faiblement disponibles. En utilisant des résultats obtenus au cours de différentes thèses de doctorat passées, il a été possible de proposer aux étudiants un « atelier» pour jouer avec des fonctions et se confronter à la problématique de l'autonomie énergétique ou encore d'une alimentation de tension continue, autonome, d'objets peu consommateurs qui seront appelés ici des capteurs autonomes.

On notera qu'il n'est pas possible au cours d'un atelier d'éviter les questionnements des étudiants périphériques au sujet pratique. Qu'il s'agisse d'un moyen de détourner l'attention de l'enseignant ou d'interrogations légitimes, un atelier doit garder un cap qui est celui de son objectif pédagogique sans pour autant fermer la porte aux idées. En particulier, l'atelier qui est décrit dans ce document, a été testé par trois types de publics : des étudiants d'IUT, des étudiants en cycle ingénieur et des doctorants. Leur intérêt technique s'exprime différemment mais l'évocation de l'Internet des Objets débouchent immanquablement sur la question du "self quantified", de la finalité réelle des objets interconnectés et de la sécurité des personnes comme des données. Sans occuper cours à ces questions qui méritent qu'un pendant en Sciences Humaines accompagnent les cours dans ce domaine, on peut cadrer la discussion en la ramenant sur le terrain des fonctions à considérer et de leur coût en énergie. L'atelier proposé veut concentrer l'activité et la réflexion de l'étudiant sur le vecteur énergie au sein des objets connectés. 
La partie 2 décrit les objectifs simples de l'atelier et sa structure est évoquée dans la partie 3. Le déroulement de l'atelier est illustré à travers 3 exemples. Un exemple très simple (partie 4) reprend les conseils donnés par les enseignants sans réel apport de la part des quelques étudiants qui ont testé l'atelier en avance sur son introduction dans des cursus. Un second exemple (partie 5) montre que des étudiants qui ont préparé l'atelier et joué le jeu d'une proposition, peuvent la mettre en oeuvre, de manière plus ou moins heureuse. Un troisième exemple (partie 6) est issu d'étudiants en formation doctorale mais non issue d'une formation EEA. Cet exemple montre que l'approche de type lego voulue pour cet atelier permet de dépasser certaines difficultés techniques pour permettre à des étudiants d'accéder à une réalisation pratique d'une solution qu'ils ont eux-mêmes pensée. Des conclusions sur une première évaluation de l'atelier sont données en partie 7.

\section{Objectifs de l'atelier}

Comme l'atelier n'a pas vocation d'aller en profondeur sur les tenants-et-aboutissants des fonctions mises en oeuvre pour récupérer de l'énergie ambiante, la durée ne peut pas excéder $4 \mathrm{~h}$ à $8 \mathrm{~h}$. C'est une durée suffisante pour un étudiant pour parvenir à construire une solution à la façon d'un lego, de pratiquer des mesures de performances et de réserver un temps de discussion finale.

Les objectifs de l'atelier pour l'étudiant sont simples :

- Sur la base des spécifications en alimentation d'un capteur (à faible consommation), réfléchir à une solution d'alimentation en tension, autonome, à partir de micro-générateurs.

- Les données à manipuler à propos de micro-générateur doivent mener à les caractériser. L'étudiant réfléchit à la caractérisation dont il a le plus besoin et à la manière de la mettre en oeuvre.

- Comme toute construction, l'étudiant doit adopter une certaine méthodologie pratique, notamment pour débogguer son câblage.

- Avec une solution entre les mains, l'étudiant joue assez librement des scénarios de consommation et les plus rapides veulent tenter un test avec un capteur réel.

- L'étudiant est invité à résumer en quelques lignes d'anglais ce que l'atelier lui a apporté sur le fond et sur la forme et il est invité à poser 3 questions. 


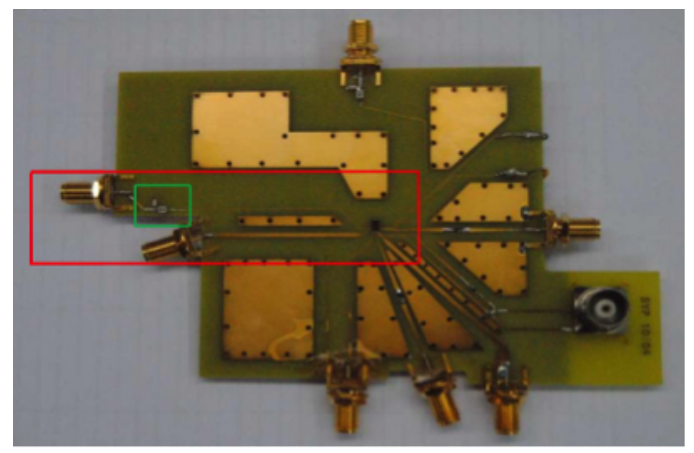

Figure 1 - Carte de structures de redressement, à interfacer avec des antennes pour former des rectenna et tenter de récupérer de l'énergie à partir du champ électromagnétique ambiant. Le rectangle vert point sur une structure à une diode en composants discrets. Le rectangle rouge est une combinaison de structures de redressement intégrées et en composants discrets

La phase finale de discussion collégiale part des questions des étudiants. On s'attachera aux plus pertinentes et/ou à celles qui intéressent une majorité d'étudiants. L'étudiant doit compléter ses notes d'une liste d'éléments techniques à approfondir.

\section{Structure de l'atelier}

Les moyens mis à disposition des étudiants sont

- des micro-générateurs de 2 types (biopiles microbiennes et rectenna),

- des composants ou macro-composants pour tester des convertisseurs de tension ou de courant à découpage de tous types,

- tous les composants électroniques standard permettant un report simple sur plaquette de type ProtoBoard,

- des sources de tension/courant de laboratoire pour les caractérisations,

- des instruments de mesure de tension et de courant (multi-mètre, sondes, oscilloscope, carte d'acquisition analogique/numérique).

La figure 1 illustre une platine embarquant 3 types de circuits redresseurs, à connecter à une antenne pour former une rectenna. Les antennes sont de type patch et constituent une problématique de l'atelier, mais non abordée car nécessitant des moyens de mise en oeuvre plus compliqués et gourmands en temps. 


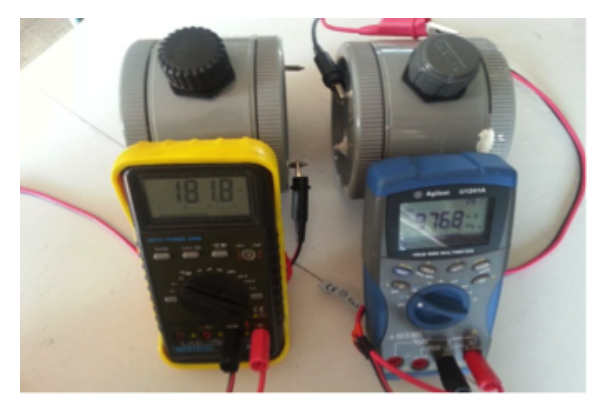

FiguRE 2 - Réacteurs de biopiles microbiennes, inoculés et accessibles uniquement par leurs sorties électriques

Les différents types de circuits permettent de s'adapter à tout public étudiant. La forme la plus intégrée constitue bien évidemment l'objet de discussion ultime puisque ceci débouche sur des questions technologiques et de conception.

La figure 2 montre des réacteurs de biopiles microbiennes. Des documents transmis au préalable aux étudiants en expliquent la constitution et brièvement le principe. Le lien entre électricité et monde vivant suscite beaucoup de question à la fin de l'atelier.

En amont du déroulement de l'atelier, l'étudiant est en charge d'évaluer la consommation de divers capteurs dont il lui est donné référence. En particulier l'étudiant doit faire la différence entre les consommations électriques représentatives de certaines activités des capteurs de la consommation moyenne. En considérant des profils de mission représentatifs, l'étudiant évalue numériquement (sous Matlab ou MathCad) des bilans énergétiques pour spécifier des sources autonomes en énergie.

Toujours au rang de la préparation, l'étudiant reçoit une littérature minimaliste qui présente les grands traits des deux types de micro-générateurs qui seront à sa disposition (biopiles microbiennes et rectenna en champ électromagnétique libre). Il doit évaluer les capacités de ces transducteurs en termes de tension maximale, courant maximal et puissance maximale, donc établir un bilan en énergie sur un temps donné. Il rapprochera ces chiffres des spécifications établies précédemment. Enfin l'étudiant doit réfléchir à un protocole p our c aractériser en pratique les micro-générateurs.

Il est sans doute bon de préciser que dans ce contexte, rares sont les étudiants rencontrés qui n'ont pas tout de suite mis en évidence que la boitille microbienne se rapprochait le plus de l'idée qu'ils ont d'un réservoir d'énergie et que la rectenna n'a pas suscité grand intérêt. La mise en oeuvre d'une rectenna est plus compliquée (seuls un doctorant beta-testeur s'en est emparé, alors que sa formation et sa 
spécialité n'ont rien à voir avec l'électronique radio-fréquence ni les antennes).

En séance, les étudiants débutent par la phase de caractérisation des sources de manière à laisser du temps à l'enseignant pour venir discuter de la solution de grappillage imaginée par l'étudiant. L'enseignant adopte alors deux positions :

1. la proposition de l'étudiant témoigne d'un travail sérieux de préparation et reçoit un argumentaire cohérent : l'étudiant est invité à la mettre en oeuvre. L'étudiant sera épaulé autant que faire se peut;

2. l'étudiant a survolé la problématique ou mal compris l'objectif ou vient avec une proposition maigre : l'enseignant lui propose un plan de repli sous la forme d'une solution simple, minimaliste mais sans risque d'échec. L'étudiant sera moins aidé.

$\mathrm{Au}$ trois-quart du temps de séance, l'étudiant est invité à procéder à l'évaluation des performances de sa construction, en l'état. L'expérience en beta-test de l'atelier a montré que 30 minutes sont largement suffisantes pour cette phase et la consignation de notes.

Cinq minutes sont accordées à l'étudiant pour coucher sur le papier 3 questions que lui inspirent son travail, l'atelier au sens général, la problématique de la récupération d'énergie ou autre chose en lien avec l'autonomie énergétique d'objets communicants.

La phase de debrie ing est limitée à 30 minutes mais pourrait durer davantage car des questions pertinentes surgissent et la liste des éléments scientifiques et techniques en lien avec l'atelier peut rapidement s'allonger en fonction du public d'étudiants.

La structure de l'atelier permet d'ajuster le contenu et l'objectif en fonction du public. L'atelier peut même s'adresser à des lycéens.

\section{Exemple typique}

La figure 3 montre un résultat réalisé par un étudiant issu d'un cursus ingénieur en « électronique intégrée » dans le cadre de sa formation doctorale obligatoire. Utilisant un transformateur à deux enroulements et un transformateur à trois enroulements, l'étudiant a souhaité réaliser un oscillateur de type Armstrong qu'il a extrait de la littérature. Celui-ci est commandé par un transistor P-JET discret, vu dans les cours d'électronique élémentaire. Une structure lyback est tout droit issue des cours d'électronique de puissance (ou switch-mode power supply comme on le voit dans beaucoup de cursus d'ingénierie proche de l'électronique). Dans cet exemple, l'étudiant avait préalablement vu la structure flyback en fonctionnement 


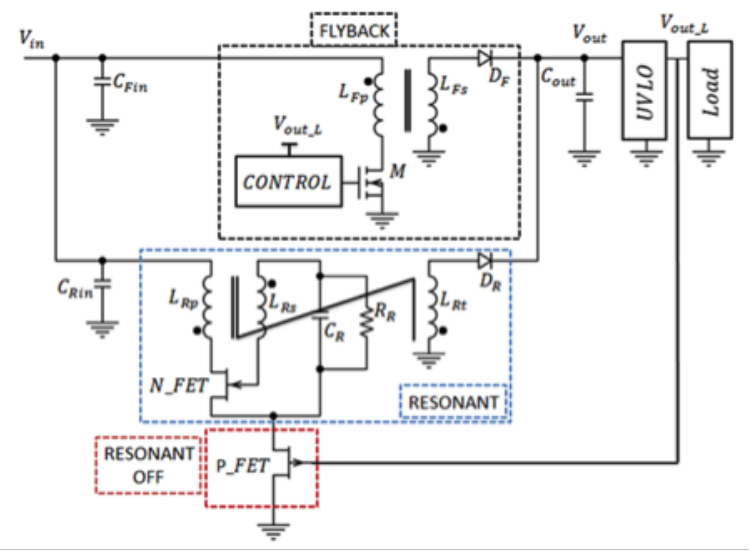

Figure 3 - Schéma de conversion d'énergie en aval d'une rectenna

normal, dit de conduction continue. Il n'envisageait pas d'autre mode de conduction et c'est pourtant l'information essentielle qu'il retiendra de l'atelier : le mode de conduction discontinue et sa propension à permettre l'adaptation de l'impédance de la charge à celle de la rectenna (non représentée sur le schéma).

Un test réalisé avec un jeu de résistances pour émuler la consommation électrique d'un capteur, montre sur la figure 4 la tension aux bornes du condensateur $C_{\text {out }}$ dans le schéma de la figure 3. Au début l'oscillateur commandé par le composant P-JFET réagit à une très faible tension d'entrée $(50 \mathrm{mV})$ et charge le condensateur jusque $0.9 \mathrm{~V}$, suffisant pour bloquer le P-JFET. La structure flyback prend le relais pour atteindre environ $3 \mathrm{~V}$ de tension de sortie. Ceci illustre une phase de démarrage à froid, qui constitue un sujet de recherche en soi. Durant cette phase la résistance de sortie, très grande, indique l'absence de consommation de la part d'un éventuel capteur (ou son énergie de maintien en veille). L'oscillogramme montre ensuite comment la tension de sortie du système est perturbée quand une charge est connectée (c'est-à-dire une résistance de valeur significative). Sous $1 M \Omega$, tout va bien; sous $100 k \Omega$, mieux vaut limiter le temps de connexion et sous $10 k \Omega$, il y a écroulement de l'alimentation. Ce relevé expérimental permet à l'étudiant de réagir sur sa préparation à l'atelier et son évaluation du bilan énergétique entre récupération et consommation. Cet exemple montre une solution d'alimentation peu performante en l'état mais ouvre les yeux de l'étudiant sur des réalités en terme de tensions faibles, d'impédance, de rendement... En alimentant la rectenna par un champ électromagnétique un peu plus grand, l'étudiant peut rechercher une réponse à sa solution d'alimentation pour la rendre compatible avec un capteur réel et faire un test pour se persuader de la viabilité de la solution (même perfectible). 


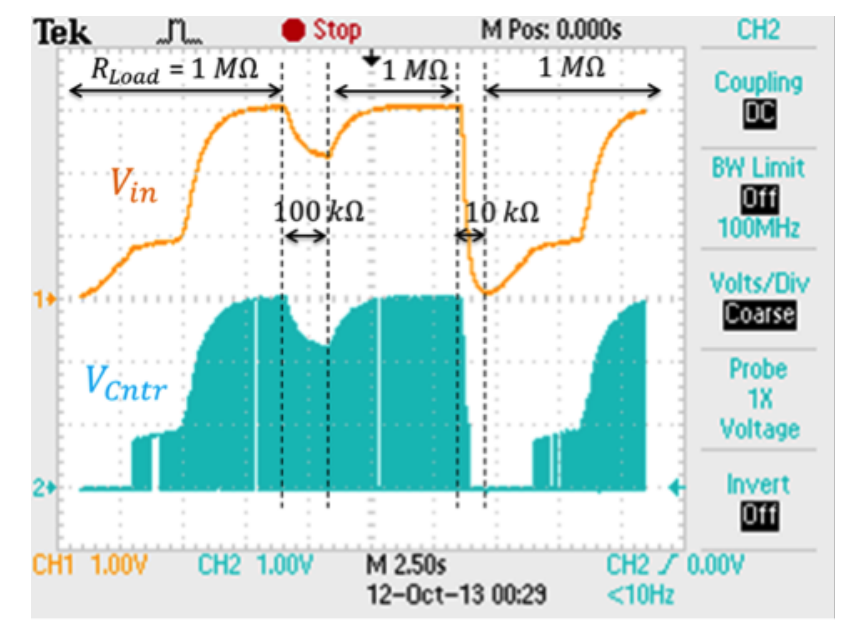

Figure 4 - Relevé d'ondes sur une construction de solution d'alimentation électrique à base de rectenna.

Sur la base du choix précédent, en ajoutant un contrôleur analogique à base de transistors MOSFETs à faible tension de seuil, l'enseignant peut montrer à l'étudiant qu'une optimisation supplémentaire permet d'atteindre des performances quasi acceptables où les pertes intrinsèques de l'alimentation, lorsque la structure flyback est en fonctionnement, sont de l'ordre de $30 \mu \mathrm{W}$ pour une énergie ambiante électromagnétique disponible de $50 \mu W$ !

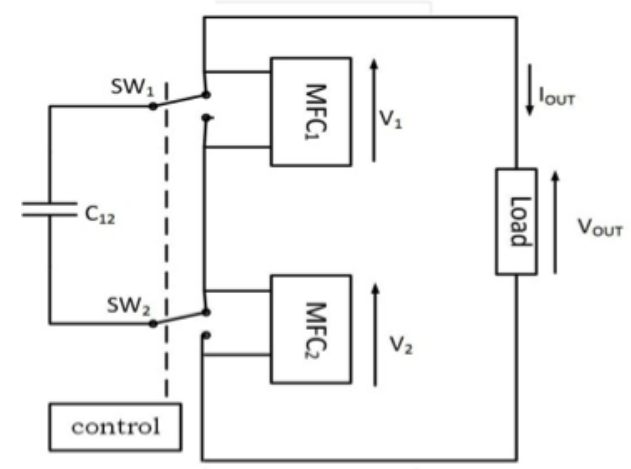

Figure 5 - Principe de l'équilibrage actif d'un lot de biopiles microbiennes en série 


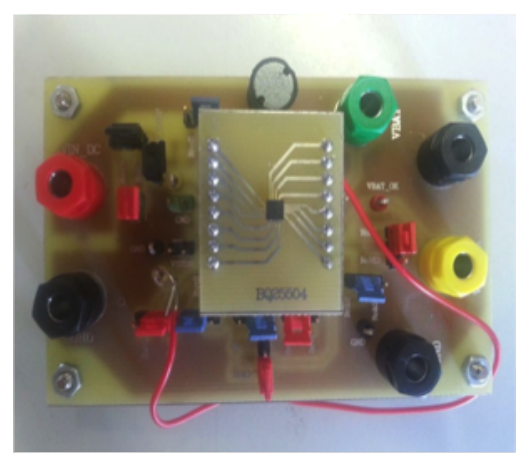

Figure 6 - Circuit intégré comportant des interrupteurs analogiques à faible perte

\section{$5 \quad$ Exemple avancé}

Une biopile microbienne n'est pas soumise comme une rectenna, aux aléas de champ électromagnétique ambiant. La production d'électricité est plus constante, quoique toujours faible $(<300 \mathrm{mV}$ pour un réacteur de bonne taille, à son point de maximum de puissance électrique). Dans ce cadre, les étudiants sont guidés non pas vers les convertisseurs élévateurs de tension mais sur la mise en série de microgénérateurs. L'étudiant est vite confronté au problème commun avec les cellules photovoltaïques : le renversement de tension. Ce phénomène conduit à ne disposer que d'une petite part de l'énergie totale disponible sur les réacteurs, pris séparément. L'atelier permet alors à l'étudiant d'expérimenter des solutions d'équilibrage actif. La figure 6 montre le principe d'équilibrage à base de capacités commutées.

La conversion de tension à base de capacités commutées est enseignée dans de nombreuses formations. Il s'agit de principe de base. L'atelier doit donc proposer une approche un peu différente de l'application de ce principe de conversion. Les biopiles se livrent mieux que les rectennas à la mise en série puisqu'il faut alors disposer d'antennes différentielles, ce qui entraînerait l'atelier trop loin de son objectif.

La figure 7 montre un relevé pris dans les notes d'un étudiant qui s'est attaché à mesurer scrupuleusement les caractéristiques statiques de 2 biopiles mises en série et dont la plus fragile présente une inversion de tension à fort courant de sortie. Le schéma de principe de la figure 5 peut facilement être mis en oeuvre d'autant qu'un macro-composant est disponible (figure 6) pour faciliter l'implémentation des interrupteurs analogiques. Ceci rend possible la réalisation par prototype rapide de manière à éviter le recours à un circuit imprimé, qui même avec une solution de gravure, grèverait en temps la session d'atelier. De la même manière, un générateur d'horloge très faible consommation est disponible sous la même forme que la platine 


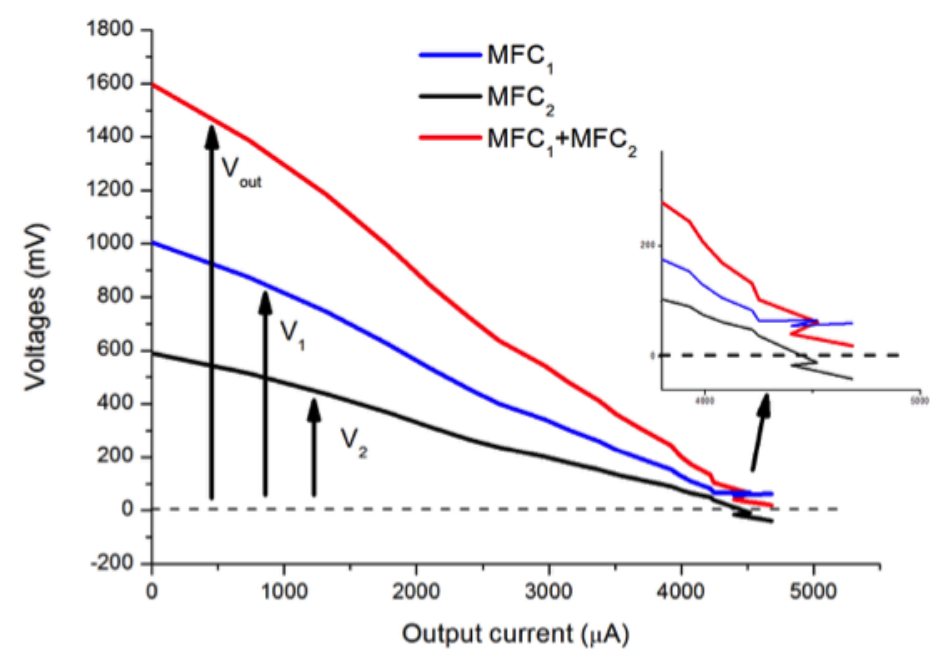

FiguRE 7 - Relevé de caractéristiques statiques de biopiles microbiennes montrant l'effet de renversement de tension aux bornes de la boitille la plus fragile

d'expansion de la figure 6 .

Un étudiant d'IUT, beta-testeur, a voulu pousser l'analyse des caractéristiques de biopiles en remarquant que les courbes de la figure 7 correspondent peu ou prou à celle d'un générateur non-idéal de tension. ayant bien préparé la séance et notamment ouvert un des documents disponibles ( [2]) a voulu discuter les paramètres du schéma équivalent de la figure 8. L'enseignant doit à ce moment-là intervenir pour éviter que l'atelier ne se transforme en une séance d'identification de paramètres, délicate à ce stade et avec les réacteurs proposés. Par contre, le même étudiant a su compléter ses notes d'une simulation réalisée en VHDL-AMS pour comparer ses relevés expérimentaux à une approche d'évaluation plus théorique. La figure 9 démontre qu'avec les moyens assez rustres mis à disposition des étudiants, ceux-ci peuvent mener une réflexion constructive et pertinente. La figure 10, également issue des notes du même étudiant, évalue une sorte d'efficacité de l'équilibrage (jusqu'où est-ce utile). Même si le terme d'efficacité est mal venu en la circonstance, et outre la démonstration de l'interêt d'équilibrer des biopiles microbiennes en série, l'étudiant a poursuivi son travail par une simulation, certes simple, mais qui prouve l'impact positif de l'atelier : sinon aucun étudiant ne serait tenté de poursuivre et compléter son travail. 


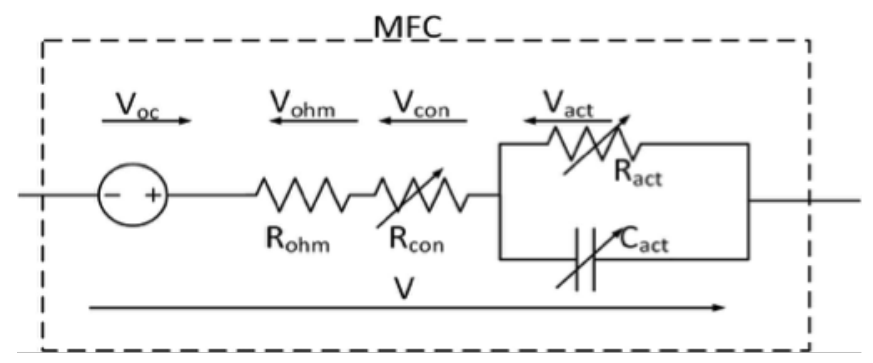

FigurE 8 - Schéma électrique équivalent au comportement en tension d'une biopile microbienne

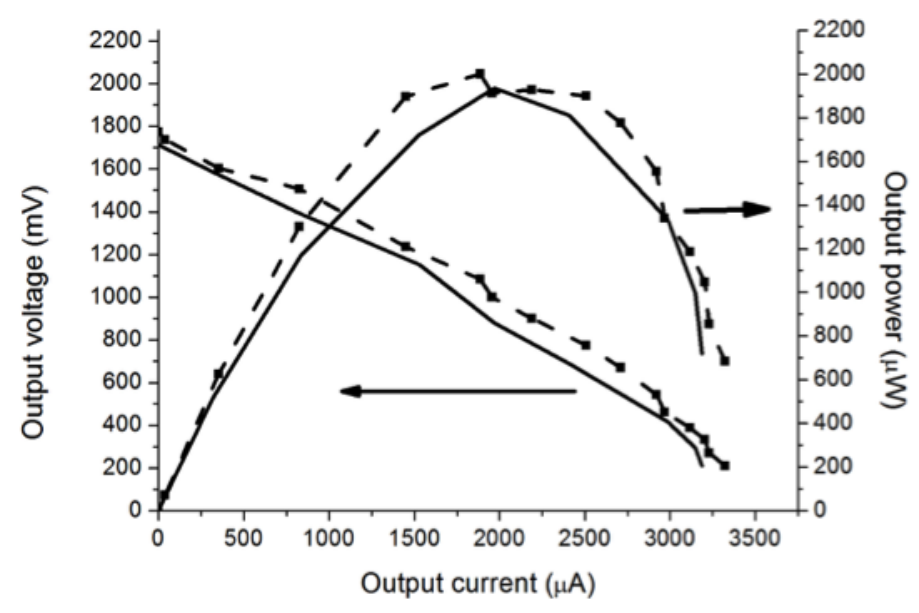

Figure 9 - Comparaison de relevés expérimentaux avec une simulation simple du schéma de principe de la figure 5 


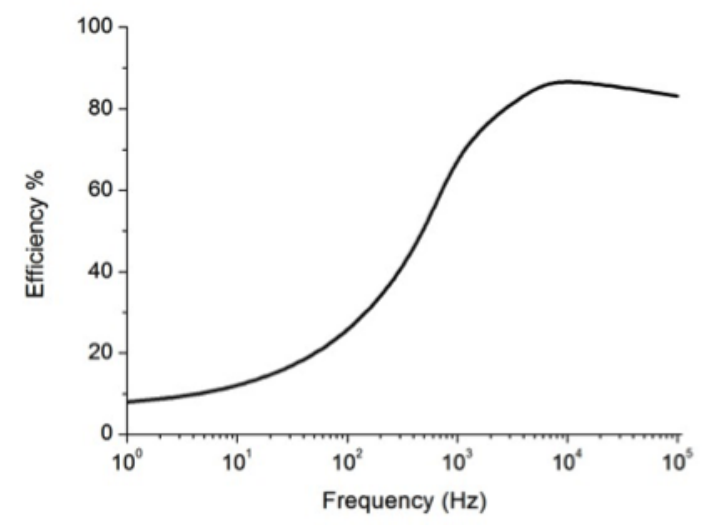

Figure 10 - Evaluation de l'efficacité de l'équilibrage actif

\section{Exemple encourageant}

Deux étudiants en formation doctorale et s'intéressant à des aspects biologiques autour des bactéries ont été intéressés par le concept de l'atelier et choisi de s'y confronter. Leur formation, loin de l'EEA, ne les prédispose pas à mener à bien l'objectif proposé par l'atelier. En fait, la préparation a permis aux étudiants de comprendre certains éléments liés à la récupération d'énergie. Les étudiants ont indiqué avoir complété leur information d'articles tirés des Techniques de l'Ingénieur. Quoiqu'il en soit, les étudiants ont proposé de pousser l'analyse de la mise en série de biopiles avec un lot de 4 biopiles. Ils ont donc prévu de câbler le schéma de principe de la figure 11 .

$\mathrm{Au}$ bout de 3 heures acharnées de câblage, test, correction, les étudiants avaient devant eux le spectacle de la figure 12. Il est sorti des résultats de ce câblage mais la figure montre les limites de l'atelier, et en tout cas, de la technologie proposée aux étudiants pour donner libre cours à leur expérimentation.

Des étudiants, non issus d'un cursus EEA, mais curieux de concepts connexes à leurs préoccupations doctorales, ont tout de même réussi (certes en suivant une recette) à bobiner un transformateur (figure 14) pour réaliser la structure flyback pour la conversion de l'énergie issue du lot de biopiles en série (bloc convertisseur DC/DC de la figure 11, schéma de la figure 13). Le composant S-882Z embarque un convertisseur à capacités commutées pour élever la tension en sortie du lot de biopiles. Dès que la tension de sortie atteint $1,2 \mathrm{~V}$, l'oscillateur TS3001 démarre et met la structure flyback en fonction. 


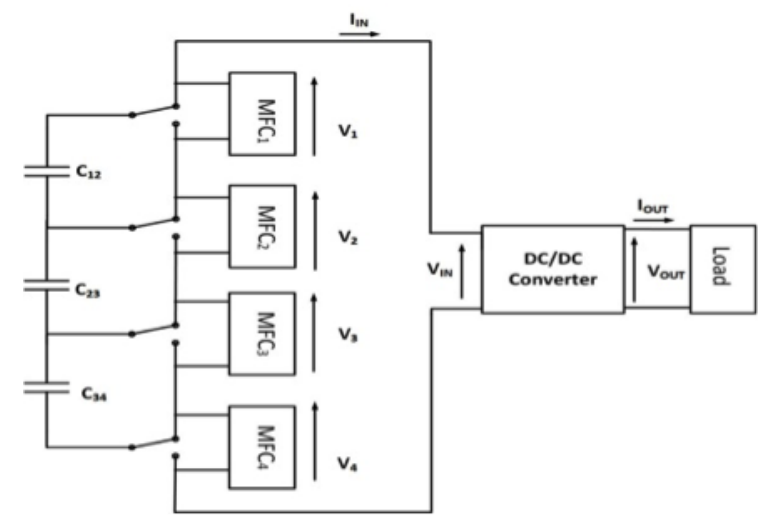

FiguRE 11 - Schéma de principe avec 4 biopiles en série

TABle 1 - Tableau de mesures dans un cahier d'étudiant

\begin{tabular}{|c|c|c|c|}
\hline & $V_{o c}[\mathrm{mV}]$ & MPP $[\mu \mathrm{W}]$ & $\eta[\%]$ \\
\hline Theoretical association & 1673 & 2255.9 & 100 \\
\hline No balancing & 1610 & 1735 & 75 \\
\hline Balancing & 1715 & 2041 & 90 \\
\hline
\end{tabular}

La phase de debriefing avec ces doctorants a été un peu particulière. Les étudiants n'avaient plus besoin d'être convaincus de l'intérêt de la récupération d'énergie ni des difficultés à gérer de faible flux d'énergie. Leur curiosité pour l'électronique, que finalement i ls c ommençaient à m ettre en o euvre p our la p remière fois, les a poussé à demander une sorte de correction. Collégialement avec l'enseignant, les étudiants ont corrigé leur circuit et ont pu produire des résultats plus pertinents.

Les étudiants en formation doctorale ont pris l'habitude de consigner davantage de faits et observations dans leur cahier de manipulation et le tableau ci-dessus montre qu'ils ont mené une analyse sérieuse des performances de leur solution de valorisation électrique de biopiles. La tension $V_{o c}$ caractérise la biopile à vide : c'est ainsi que les étudiants cherchent à évaluer le point de fonctionnement de chaque boitille dans l'association série, à partir de leur caractéristique statique (comme sur la figure 9). Le relevé MPP correspond au maximum power point de l'association de biopiles, avec ou sans équilibrage. Enfin les étudiants ont cherché à évaluer l'intérêt de l'équilibrage en prenant une base 100 pour le cumul des puissances de chaque biopile prise séparément. Les résultats sont tout à fait réalistes et témoignent du soin des étudiants durant l'atelier. 


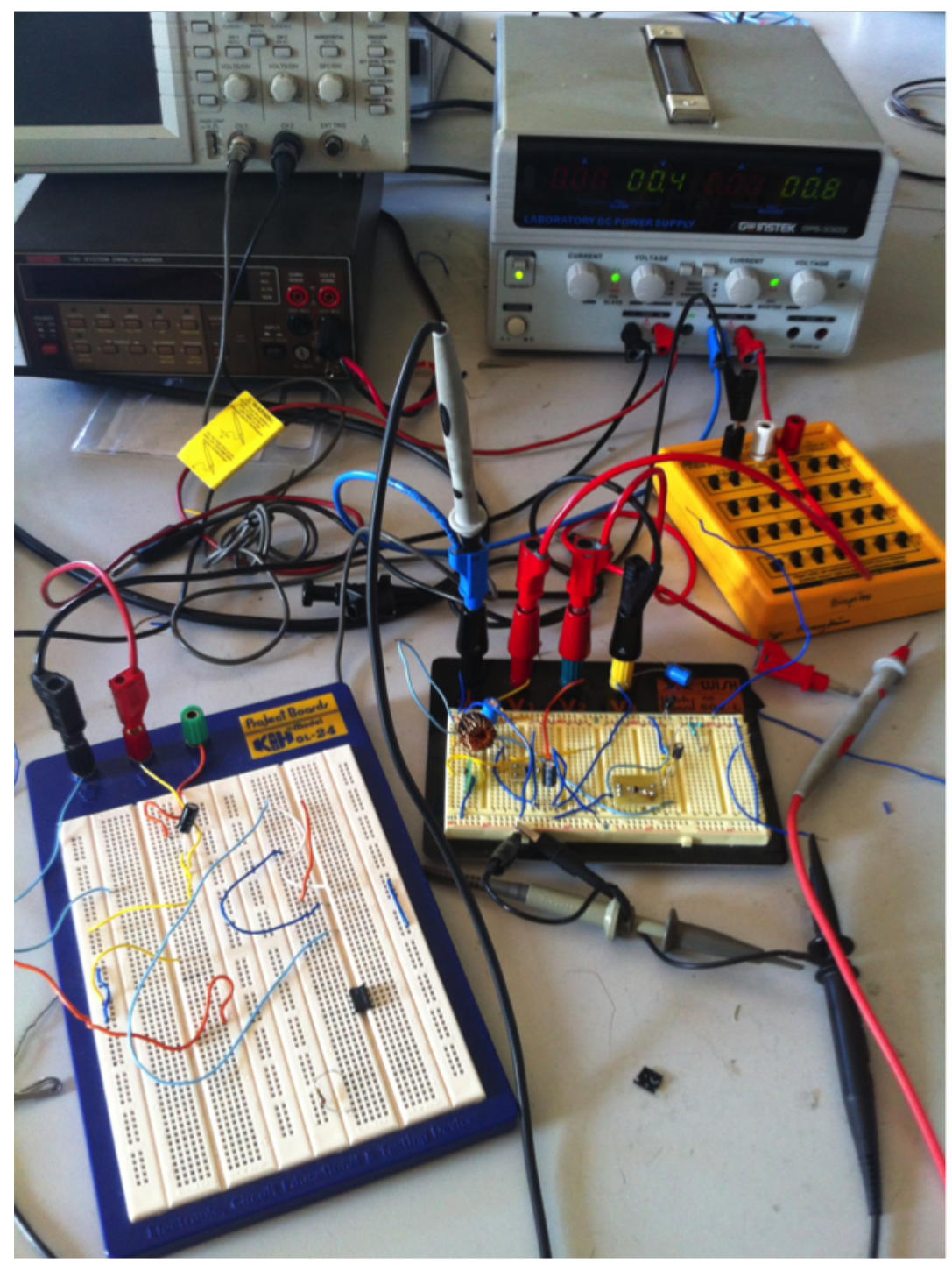

FiguRE 12 - Résultats du travail d'étudiants non formés à l'électronique en cursus initial 


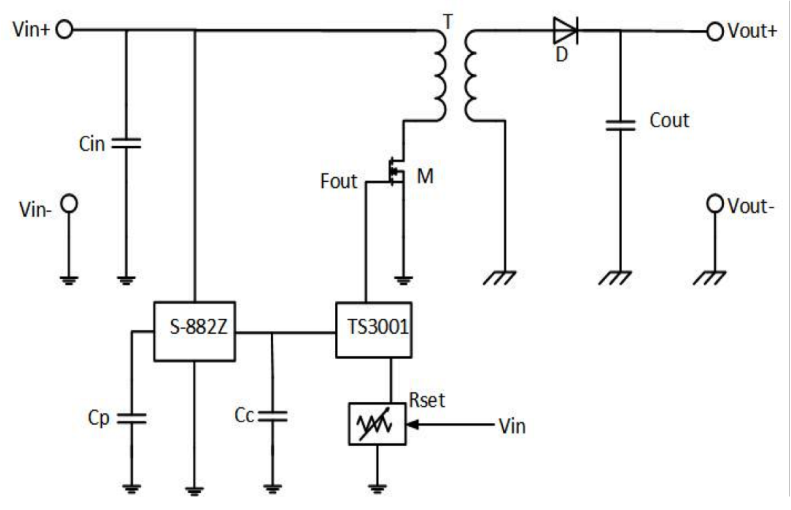

Figure 13 - Schéma avancé de conversion de tension en sortie du lot de biopiles

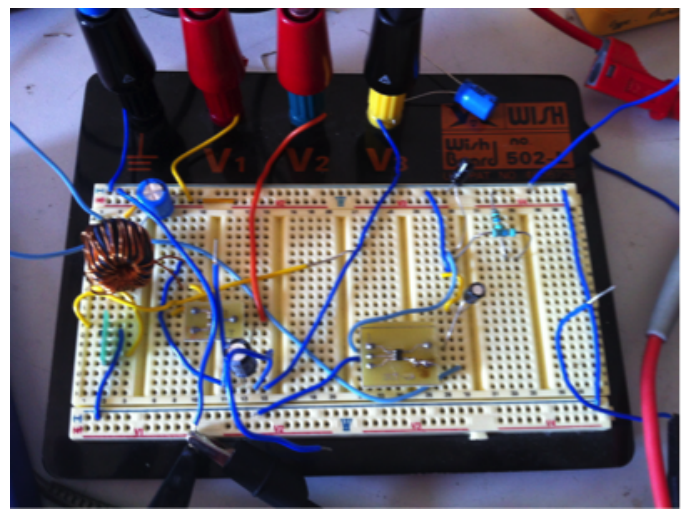

FiguRe 14 - Zoom sur un transformateur bobiné par des étudiants au cours de l'atelier 


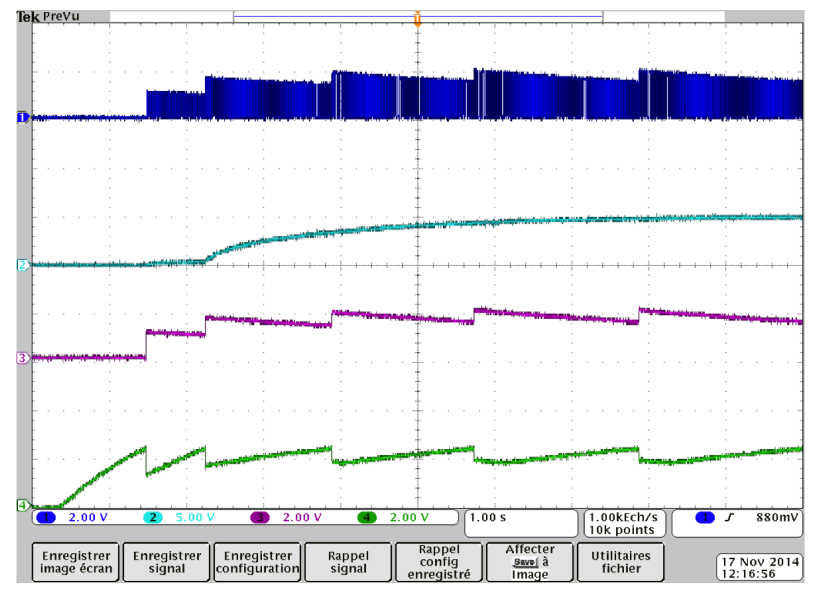

Figure 15 - Relevé d'ondes à l'oscilloscope sur un schéma dont le calage a été retouché

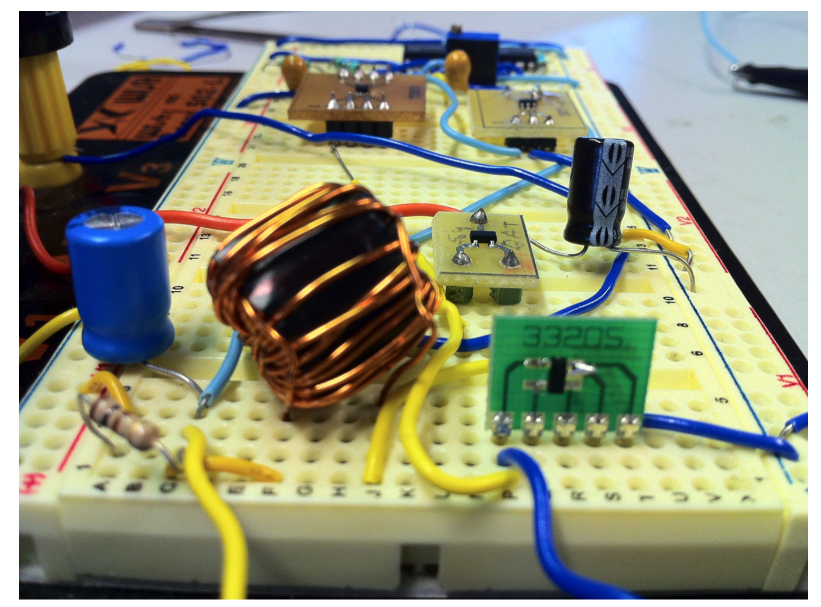

Figure 16 - Photo d'une platine propre 


\section{Première évaluation}

L'atelier a été testé par 2 étudiants d'IUT, 5 lycéens en Première S et 2 doctorants (qui ne sont pas du domaine EEA). Le format de manipulation avec des macro-blocs prêts à l'usage facilite l'appropriation de la partie pratique. Il n'en reste pas moins qu'il s'agit d'électronique et que des petits détails viennent vite limiter l'ambition de l'atelier. En particulier les étudiants pointent le manque de robustesse de l'approche par plaquette Protoboard car le câblage reste assez lâche et rapidement des fauxcontacts et des pertes de contact surviennent.

La phase de préparation n'a pas posé, semble-t'il, de problème particulier aux étudiants. C'est-à-dire que la littérature qui leur a été fournie les a aidé à réfléchir : s'approprier l'objectif, comprendre les contraintes et poser une solution, sur la base de schémas suggérés. A part un étudiant, a posteriori de l'atelier, aucun n'a spontanément pensé et mis en oeuvre une analyse du problème par la simulation. On ne peut pas tirer de généralité sur un petit groupe de beta-testeurs de l'atelier, qui plus est hétérogène en cursus, mais il s'agit d'une remarque sérieuse, qui doit pousser à motiver les étudiants, par la suite, à s'approprier une phase de simulation.

L'atelier sera proposé en 2015-2016 à des étudiants de cursus ingénieur EEA, sur la base du volontariat car hors module pour l'instant, et à des étudiants de Master comme illustration pratique des cours de tronc commun dans un parcours dédié aux systèmes embarqués communicants.

\section{Conclusion}

L'idée de l'atelier comme approche simple de concepts de récupération d'énergie semble remplir ses objectifs. Des étudiants de cursus autre que l'EEA sont parvenus à jouer avec les blocs fonctionnels pour vérifier expérimentalement une solution issue de leur réflexion sur l'équilibre entre consommation électrique et énergie "gratuite" (ambiante) disponible.

Le format de l'atelier est également bien proportionné. C'est en tout cas ce que visera à confirmer la première session avec un nombre significatif d'étudiants.

L'investissement pour les enseignants se situe sur les documents de préparation et la liste des composants disponibles pour les étudiants. En séance, il faut trouver un bon équilibre entre le soutien, le conseil et l'aide trop appuyée. C'est sans doute ce qu'on appelle du coaching. L'enseignant est par contre pris en séance par les problèmes électriques liés à l'approche de prototype rapide. Cette approche exige quelques règles simples mais qu'il ne suffit pas simplement de rappeler aux étudiants. 
Ils découvrent donc les aléas pratiques du prototypage rapide, qui perturbent le travail qui se voudrait égal à celui de l'assemblage d'un lego. La figure 16 présente un exemple de câblage au plus propre de ce que les étudiants, peu rompus à la technique, peuvent produire. Il faut donc en tenir compte car les performances du système obtenues en pratique, en sont affectées. Il faut éviter que les étudiants partent avec des acquis en demi-teinte à cause de cet aspect de prototypage rapide. Sans doute faudra-t-il soit guider davantage les étudiants $\mathrm{du}$ point de vue pratique ou considérer une autre forme technique pour atteindre une approche plus proche du lego.

\section{Références}

[1] J. Verdier, T. Risset, K. Market, G. Salagnac, L. Morel, D. Tournier, L.V. Phung, F. Hutu and B. Allard, Formation par projet et opportunité d'accès à distance à des ressources pédagogiques, Actes de CETSIS, Caen, 2013.

[2] J. Larminie and A. Dicks, Fuel cell systems explained, 2nd edn, New York : Wiley, 2003

Bruno Allard est enseignant à l'INSA de Lyon, Professeur des Universités au Département de Génie Electrique et chercheur au laboratoire Ampère, UMR CNRS 5005. Il est responsable d'enseignement en électronique mixte analogique-numérique. Il est Directeur du Pôle de Lyon du GIP CNFM.

Jacques Verdier est enseignant à l'INSA de Lyon, Maître de Conférences au Département de Génie Electrique et chercheur au laboratoire Ampère, UMR CNRS 5005. Il est responsable d'enseignement en électronique radio-fréquence. Il est DirecteurAdjoint du Pôle de Lyon du GIP CNFM. 